\title{
Ground-state properties of the Falicov-Kimball model with correlated hopping in two dimensions
}

\author{
Pavol Farkašovský and Natália Hudáková \\ Institute of Experimental Physics, Slovak Academy of Sciences \\ Watsonova 47, 04353 Košice, Slovakia
}

\begin{abstract}
A new numerical method, recently developed to study ground states of the Falicov-Kimball model (FKM), is used to examine the effects of correlated hopping on the ground-state properties of this model in two dimensions. It is shown that the ground-state phase diagram as well as the picture of metalinsulator transitions found for the conventional FKM (without correlated hopping) are strongly changed when the correlated hopping term is added. The effect of correlated hopping is so strong that it can induce the insulator-metal transition, even in the strong-coupling limit, where the ground states of the conventional FKM are insulating for all $f$-electron densities.
\end{abstract}

PACS numbers.:75.10.Lp, 71.27.+a, 71.28.+d, 71.30.+h 


\section{Introduction}

Since its introduction in 1969, the FKM [1] has become an important standard model for a description of correlated fermions on a lattice. The model was originally proposed to describe metal-insulator transitions and has since been investigated in connection with a variety of problems such as binary alloys [2], the formation of ionic crystals [3], and ordering in mixed-valence systems [4]. It is the latter language we shall use here, considering a system of localized $f$ electrons and itinerant $d$ electrons coupled via the on-site Coulomb interaction $U$. The Hamiltonian of the spinless FKM is

$$
H=\sum_{i j} t_{i j} d_{i}^{+} d_{j}+U \sum_{i} f_{i}^{+} f_{i} d_{i}^{+} d_{i}+E_{f} \sum_{i} f_{i}^{+} f_{i}
$$

were $f_{i}^{+}, f_{i}$ are the creation and annihilation operators for an electron in the localized state at lattice site $i$ with binding energy $E_{f}$ and $d_{i}^{+}, d_{i}$ are the creation and annihilation operators for an electron in the conduction band. The conduction band is generated by the hopping matrix elements $t_{i j}$, which describe intersite transitions between the sites $i$ and $j$. Usually it is assumed that $t_{i j}=-t$ if $i$ and $j$ are nearest neighbors and $t_{i j}=0$ otherwise (the conventional FKM), however, in what follows we consider a much more realistic type of hopping, so for the moment we leave it to be arbitrary.

Recent theoretical works based on exact numerical and analytical calculations showed that the FKM, in spite of its relative simplicity, can yield the correct physics for describing of such fundamental phenomena as valence-change transitions, metalinsulator transitions, crystallization, charge ordering, etc. For example, it was found that the spinless FKM, in the pressure induced case, can describe both types of intermediate-valence transitions observed experimentally in rare-earth compounds: a discontinuous insulator-insulator transition for sufficiently strong interactions [5] and a discontinuous insulator-metal transition for weak interactions [6]. In addition, at nonzero temperatures this model is able to provide the qualitative explanation for anomalous large values of the specific heat coefficient and for extremely large changes of electrical conductivity []] found in some intermediate-valence compounds (e.g., in $\mathrm{SmB}_{6}$ ). Moreover, very recently the spin-one-half version of the FKM has 
been used to describe a discontinuous intermediate-valence transition (accompanied by a discontinuous insulator-metal transition) in $\mathrm{SmS}$ [8] as well as for a description of an anomalous magnetic response of the Yb-based valence-fluctuating compounds [9].

On the other hand it should be noted that the model Hamiltonian (1) neglects all nonlocal interaction terms, and thus it is questionable whether above mentioned results persist also in more realistic situations when nonlocal interactions will be turned on. An important nonlocal interaction term obviously absent in the conventional FKM is the term of correlated hopping, in which the $d$-electron hopping amplitudes $t_{i j}$ between neighboring lattice sites $i$ and $j$ depend explicitly on the occupancy $\left(f_{i}^{+} f_{i}\right)$ of the $f$-electron orbitals. To examine effects of this term on ground-state properties of the two-dimensional FKM we choose the following form for the nearest-neighbor matrix elements

$$
\tilde{t}_{i j}=t_{i j}+t_{i j}^{\prime}\left(f_{i}^{+} f_{i}+f_{j}^{+} f_{j}\right)
$$

which represent a much more realistic type of electron hopping than the conventional hopping.

Thus the spinless FKM in which the effects of correlated hopping are included can be written as

$$
H=\sum_{<i j>} t_{i j} d_{i}^{+} d_{j}+\sum_{<i j>} t_{i j}^{\prime}\left(f_{i}^{+} f_{i}+f_{j}^{+} f_{j}\right) d_{i}^{+} d_{j}+U \sum_{i} f_{i}^{+} f_{i} d_{i}^{+} d_{i}+E_{f} \sum_{i} f_{i}^{+} f_{i} .
$$

The first term of (3) is the kinetic energy corresponding to quantum mechanical hopping of the itinerant $d$-electrons between the nearest-neighbor sites $i$ and $j$. The second term is just the correlated hopping term discussed above. The third term is the on-site Coulomb interaction between the $d$-band electrons with density $n_{d}=$ $\frac{1}{L} \sum_{i} d_{i}^{+} d_{i}$ and the localized $f$-electrons with density $n_{f}=\frac{1}{L} \sum_{i} f_{i}^{+} f_{i}$, where $L$ is the number of lattice sites. The last term stands for the localized $f$ electrons whose sharp energy level is $E_{f}$.

Since in this spinless version of the FKM without hybridization the $f$-electron occupation number $f_{i}^{+} f_{i}$ of each site $i$ commutes with the Hamiltonian (3), the $f$ electron occupation number is a good quantum number, taking only two values: $w_{i}=1$ or 0 , according to whether or not the site $i$ is occupied by the localized $f$ electron. Therefore the Hamiltonian (3) can be written as 


$$
H=\sum_{<i j>} h_{i j}(w) d_{i}^{+} d_{j}+E_{f} \sum_{i} w_{i}
$$

where $h_{i j}(w)=\tilde{t}_{i j}(w)+U w_{i} \delta_{i j}$ and

$$
\tilde{t}_{i j}(w)=t_{i j}+t_{i j}^{\prime}\left(w_{i}+w_{j}\right)
$$

Thus for a given $f$-electron configuration $w=\left\{w_{1}, w_{2} \ldots w_{L}\right\}$ the Hamiltonian (4) is the second-quantized version of the single-particle Hamiltonian $h(w)$, so the investigation of the model (4) is reduced to the investigation of the spectrum of $h$ for different configurations of $f$ electrons. Since the $d$ electrons do not interact among themselves, the numerical calculations should precede directly in the following steps. (i) Having $w=\left\{w_{1}, w_{2} \ldots w_{L}\right\}, U, E_{f}$ and the nearest-neighbor hopping amplitudes $t$ and $t^{\prime}$ fixed, (in the following $t=-1$ and all energies are measured in units of $t$ ) find all eigenvalues $\lambda_{k}$ of $h(w)$. (ii) For a given $N_{f}=\sum_{i} w_{i}$ determine the ground-state energy $E\left(w, U, E_{f}\right)=\sum_{k=1}^{N-N_{f}} \lambda_{k}+E_{f} N_{f}$ of a particular $f$-electron configuration $w$ by filling in the lowest $N_{d}=N-N_{f}$ one-electron levels (here we consider only the case $N_{f}+N_{d}=L$, which is the point of the special interest for valence and metal-insulator transitions caused by promotion of electrons from localized $f$ orbitals $\left(f^{n} \rightarrow f^{n-1}\right)$ to the conduction band states). (iii) Find the $w^{0}$ for which $E\left(w, U, E_{f}\right)$ has a minimum. Repeating this procedure for different values of $U, t^{\prime}$ and $E_{f}$, one can study directly the ground-state phase diagram and valence transitions (a dependence of the $f$ electron occupation number on the $f$-level position $E_{f}$ ) in the FKM with correlated hopping.

A direct application of this method has been used successfully in our previous papers [5, 6] for a description of ground-state properties of the one-dimensional FKM model without correlated hopping $\left(t^{\prime}=0\right)$. It was shown that finite-size effects are negligible for a wide range of the model parameters (e.g., strong interactions) and thus results obtained on relatively small clusters $(L<30)$ can be satisfactory extrapolated to the thermodynamics limit $(L \rightarrow \infty)$. Using this method we have described satisfactory the strong-coupling phase diagram as well as the picture of valence and metal-insulator transitions in the one-dimensional spinless FKM [5] with $t^{\prime}=0$. It was found that for sufficiently large $U$ the spinless FKM undergoes only a 
few discrete intermediate-valence transitions. These intermediate-valence transitions are insulator-insulator transitions, since they are realized between the insulating ground states corresponding to the most homogeneous configurations, which are the ground states in this region [10]. Thus, there are no insulator-metal transitions in the 1-d conventional FKM for strong interactions. In the next paper [11] we have shown that this picture of valence and metal-insulator transitions is dramatically changed if the term of correlated hopping is included. One of the most important results found for the one-dimensional FKM with correlated hopping was that the correlated hopping can induce the insulator-metal transition, even in the half-filled band case $n_{d}+n_{f}=1$. In this paper we try to show that the same result holds also for the two dimensional case. Similar calculations are performed also away from the half-filled band case with the goal to examine possibilities for metal-insulator transitions in the strong-coupling limit. Another inspiration for performing these calculations was the recent paper of Wojtkiewicz and Lemanski [12], where the authors studied twodimensional FKM with correlated hopping using the combination of the perturbation expansion (up to the second order) and the method of restricted phase diagrams. They found that only a few phases form the ground-state phase diagram of the model in the strong coupling limit. For example, the ground state of the model for $E_{f}=0$ is the chessboard charge-density-wave (CDW) phase for all $0<t^{\prime}<1$. Here we show that some other configurations, (e.g., the segregated configuration) can also be the ground states of the FKM at $E_{f}=0$, thereby the ground-state phase diagram as well as the picture of metal-insulator transitions are strongly changed.

\section{The method}

Since the number of configurations that should be examined to obtain the ground state energy of the FKM grows exponentially with the system size, a direct application of the exact-diagonalization method described above is restricted to clusters up to 30 sites. In our previous papers we showed that clusters of this size are sufficient to suppress finite-size effects in one-dimension [5, 6], however, to obtain trustworthy results on the ground-state energy of the model in two dimensions one has to examine much larger clusters $(L \sim 100)$. Unfortunately, the clusters with $L>30$ are beyond 
the reach of present day computers within exact diagonalizations, and thus the only way is to compute the ground-state properties of the model by an approximate but well controlled method. Here we use the simple method based on a modification of the exact-diagonalization procedure described above. The method consists of following steps: (i) Chose a trial configuration $w=\left\{w_{1}, w_{2} \ldots w_{L}\right\}$. (ii) Having $w, U$ and $E_{f}$ fixed, find all eigenvalues $\lambda_{k}$ of $h(w)=T+U W$. (iii) For a given $N_{f}=\sum_{i} w_{i}$ determine the ground-state energy $E(w)=\sum_{k=1}^{L-N_{f}} \lambda_{k}+E_{f} N_{f}$ of a particular $f$-electron configuration $w$ by filling in the lowest $N_{d}=L-N_{f}$ one-electron levels. (iv) Generate a new configuration $w^{\prime}$ by moving a randomly chosen electron to a new position which is chosen also at random. (v) Calculate the ground-state energy $E\left(w^{\prime}\right)$. If $E\left(w^{\prime}\right)<E(w)$ the new configuration is accepted, otherwise $w^{\prime}$ is rejected. Then the steps (ii)-(v) are repeated until the convergence (for given parameters of the model) is reached. Of course, one can move instead of one electron (in step (iv)) two or more $f$ electrons, thereby the convergence of method can be improved. Indeed, tests that we have performed for a wide range of the model parameters showed that the latter implementation of method, in which $N_{0}>1$ electrons $\left(N_{0}\right.$ should be chosen at random) are moved to new positions, overcomes better the local minima of the ground-state energy. This also improves the accuracy of method.

This method was first used in our recent paper [13] to study the ground-state properties of the one and two-dimensional FKM without correlated hopping. It was found that on small and intermediate clusters $(L \sim 30)$ the method is able to reproduce exactly the ground states of the conventional FKM, even after relative small number of iterations (typically 10000 per site). For such clusters the method is only rarely stopped at the local minimum. Of course, with increasing $L$ the problem of local minima appears often. Fortunately, it can be considerably reduced by more efficient algorithm (one is discussed above) or by increasing the number of iterations. The latter case imposes, however, severe restrictions on the size of clusters than can be studied with this method $\left(L \sim 100\right.$, for $10^{6}$ iterations per site). To verify the convergence of this method for the two-dimensional FKM with correlated hopping we have performed the same calculations on the cluster of $4 \times 4$ sites, where ground states can be obtained also within the exact diagonalization calculations. Numerical results obtained for a wide range of the model parameters 
$\left(t^{\prime}=-1,-0.8, \ldots 1, U=0,0.1 \ldots 10\right)$ shoved that the exact ground-states can be again reproduced after $\sim 10000$ iterations per site.

\section{Results and discussion}

The most interesting question that arises for the FKM with correlated hopping is whether the correlated hopping term can change the ground-state phase diagram and the picture of valence and metal-insulator transitions found for the conventional FKM $\left(t=-1\right.$ and $\left.t^{\prime}=0\right)$. The nature of the ground state, its energetic and structural properties, and the correlation-induced metal-insulator transitions are subjects of special interest. For the conventional FKM these problems are well understood at least in the symmetric case $\left(E_{f}=0, n_{f}=n_{d}=1 / 2\right)$. In this case the localized $f$-electrons fill up one of two sublattices of the hypercubic lattice (the charge-densitywave state) and the corresponding ground state is insulating for all $U>0$. Thus, for the finite interaction strength there is no correlation-induced metal-insulator transition in the symmetric case.

One can expect, on the base of simple arguments, that the ground-state phase diagram of the FKM with correlated hopping will be fully different from one discussed above for the conventional FKM. Indeed, the following selection of hopping matrix amplitudes $t=-1$ and $t^{\prime}>0$ may favor the segregated configuration since the itinerant $d$ electrons have the lower kinetic energy in this state. This mechanism could lead, for example, to the instability of the CDW state that is the ground state for $t^{\prime}=0$, and thereby to a metal-insulator transition, even in the symmetric case. To examine possibilities for such a transition in two dimensions we have performed an exhaustive study of the model on $6 \times 6$ and $8 \times 8$ clusters (with periodic boundary conditions) for a wide range of parameters $t^{\prime}$ and $U$. The results of numerical calculations are summarized in Fig. 1 in the form of the $t^{\prime}-U$ phase diagram. In addition to the CDW state $w_{1}$ that is the ground state at $t^{\prime}=0$ for all nonzero $U$ we found two new phases that can be the ground states of the model, and namely, the configuration with alternating lines of occupied and unoccupied sites $w_{2}$ and the segregated configuration $w_{3}$ (see Fig. 2). Thus at nonzero $t^{\prime}$ the CDW state $w_{1}$ becomes unstable against the transition to $w_{2}$ and $w_{3}$. The transition from $w_{1}$ to $w_{2}$, as well 
as from $w_{1}$ to $w_{3}$ is the insulator-metal transition since the configuration $w_{1}$ has the finite gap $(\sim U)$ at the Fermi energy [14] for all nonzero values of $U$, while both $w_{2}$ and $w_{3}$ are metallic in the corresponding regions of stability. Thus we arrive to the very important conclusion, and namely, that the correlated hopping term can induce the insulator-metal transition, even in the half-filled band case. Another important result, confirming the crucial role of the correlated hopping term can be seen from Fig. 1, where the comprehensive phase diagram of the two-dimensional FKM with correlated hopping is presented. It is seen that the correlated hopping can destroy the CDW state, even at large values of the Coulomb interaction $(U \sim 7)$. This is an unexpected result since recent results of Wojtkiewicz and Lemanski [12 based on the combination of the perturbation expansion (up to the second order) and the method of restricted phase diagrams predicted that the ground state of the model at $E_{f}=0$ and $U$ large is the CDW state for all values of $0<t^{\prime}<1$. This discrepancy is probably due to the fact that the authors examined (as possible ground states) only a restricted set of configurations (consisting of all periodic configurations having elementary cells up to 12 sites), and the segregated configuration (that should be the ground state in this region) does not belong to this set. Another possible explanation of this discrepancy is that the second-order perturbation expansion used by authors is insufficient to describe correctly the ground-state properties of the model in this region $(U \sim 7)$.

The fact that the correlated hopping can induce metal-insulator transitions indicates that the picture of valence and metal-insulator transitions found in our previous papers within the conventional FKM [0, 6, 13] should be dramatically changed if finite values of $t^{\prime}$ will be considered. The largest changes are expected in the strong coupling limit $(U>4)$, where all ground states of the conventional FKM are insulating for both 1-d and 2-d case [5, 10, 13], while the numerical results obtained for nonzero $t^{\prime}$ show on the existence of the metallic phase, at least for $n_{f}=1 / 2$. We suppose that this important result is not restricted to the half-filled band case only, but persists also for $f$-electron densities away from this point. To verify this conjecture we have performed an exhaustive study of the model for $n_{f}=1 / 4$ on $6 \times 6,8 \times 8$ and $12 \times 12$ clusters. Our numerical calculations showed that the phase diagram of the model at $n_{f}=1 / 4$ is separated into two distinct regions. In the first region $(U<2)$ the phase 
diagram has a complex structure with the ground state apparently changing point by point at every value of the correlated hopping amplitude $t^{\prime}$ for fixed interaction strength. Unfortunately, the structure of the phase diagram in this region strongly depends on the size of cluster and thus we were not able to extrapolate satisfactory these results to the thermodynamic limit $L \rightarrow \infty$. Contrary to this case, the phase diagram exhibits a very simple structure (see inset in Fig. 1) in the opposite limit $(U>2)$. In this region only two configurations are the ground states of the FKM with correlated hopping, and namely the segregated configuration and the configuration $w_{4}$ (see Fig. 2) that has been proven to be ground state of the conventional FKM for large $U$ (see Ref. [13, 15, 16]). Since the configuration $w_{s}$ is metallic and $w_{4}$ insulating we have the correlated hopping induced metal-insulator transitions also at $n_{f}=1 / 4$. The metallic phase is stable up to $U \sim 7$ and this again confirms our conjecture that the comprehensive picture of metal-insulator transitions in the FKM with correlated hopping will be fully different from one found for the conventional FKM, especially for $U$ large. To complete this picture one has to perform similar calculations for all $f$-electron densities what is a cumbersome computational task, even on $8 \times 8$ cluster. The work on this subject is currently in progress.

In summary, the effects of correlated hopping on the ground-state properties of the FKM in two dimensions have been studied. It was shown that the groundstate phase diagram as well as the picture of metal-insulator transitions found for the conventional FKM are strongly changed when the correlated hopping term is added. The effect of correlated hopping is so strong that it can induce the insulatormetal transition, even in the strong-coupling limit, where the ground states of the conventional FKM are insulating for all $f$-electron densities.

This work was supported by the Slovak Grant Agency VEGA under grant No. $2 / 7021 / 20$. Numerical results were obtained using computational resources of the Computing Centre of the Slovak Academy of Sciences. 


\section{References}

[1] L.M. Falicov and J.C. Kimball, Phys. Rev. Lett. 22, 997 (1969).

[2] J.K. Freericks and L.M. Falicov, Phys. Rev. B41, 2163 (1990).

[3] Ch. Gruber, J.L. Lebowitz and M. Macris. Phys. Rev. B 484312 (1993).

[4] K. Ramirez, L.M. Falicov, Phys. Rev. B3, 2455 (1971).

[5] P. Farkašovský, Phys. Rev. B 51, 1507 (1995).

[6] P. Farkašovský, Phys. Rev. B 52, R5463 (1995).

[7] P. Farkašovský, Phys. Rev. B 54, 7856 (1996).

[8] P. Farkašovský, Phys. Rev. B 54, 11261 (1997).

[9] J.K. Freericks and V. Zlatic, Phys. Rev. B 58, 322 (1998); See also V. Zlatić, J. K. Freericks, R. Lemanski, and G. Czycholl, cond-mat/0107040.

[10] P. Lemberger, J. Phys. A 25, 715 (1992).

[11] P. Farkašovský and S. Gál, Phase Transitions XX, xxx (2001).

[12] J, Wojtkiewicz and R. Lemanski, cond-mat/0106200.

[13] P. Farkašovský, Eur. J. Phys. B 20, 209 (2001).

[14] Since we consider the case $N_{f}+N_{d}=L$, the Fermi level $E_{F}$ and the energy gap $\Delta$ of some configuration $w$ are given by $E_{F}=\lambda_{L-N_{f}}$ and $\Delta=\lambda_{L-N_{f}+1}-\lambda_{L-N_{f}}$, respectively.

[15] T. Kennedy, Rev. Math. Phys. 6, 901 (1994).

[16] G.I. Watson and R. Lemansky, J. Phys. Condens. Matter 7, 9521 (1995). 


\section{Figure Captions}

Fig. 1. $t^{\prime}-U$ phase diagram of the two-dimensional FKM with correlated hopping at half-filling $\left(E_{f}=0, n_{f}=n_{d}=0.5\right)$. Three different phases correspond to the CDW state $w_{1}$, the configuration with alternating lines of occupied and unoccupied sites $\left(w_{2}\right)$, and the segregated configuration $w_{3}$. The inset shows $t^{\prime}-U$ phase diagram for $n_{f}=1 / 4$ and $U>2$. Two different phases correspond to the segregated configuration and the configuration $w_{4}$ that has been proven to be ground state of the conventional FKM for large $U$.

Fig. 2. The ground-state configurations of the two dimensional FKM with correlated hopping for $n_{f}=1 / 2\left(w_{1}, w_{2}\right.$ and $\left.w_{3}\right)$ and $n_{f}=1 / 4\left(w_{4}\right)$. 


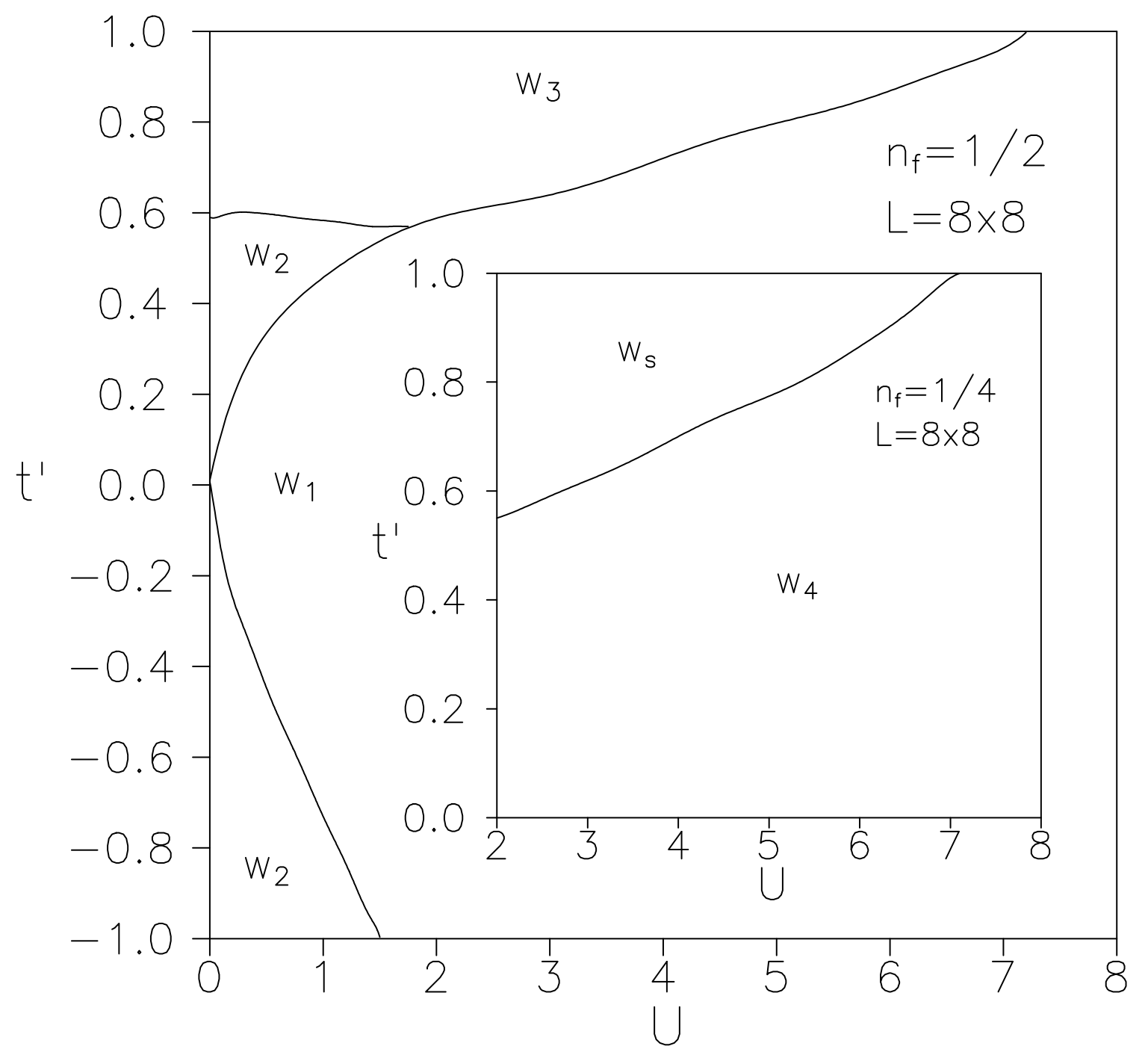




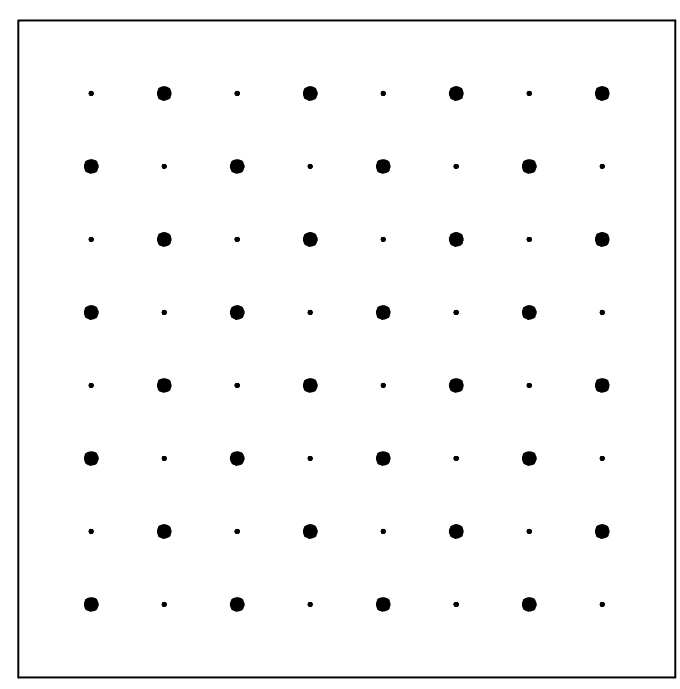

$w_{1}$

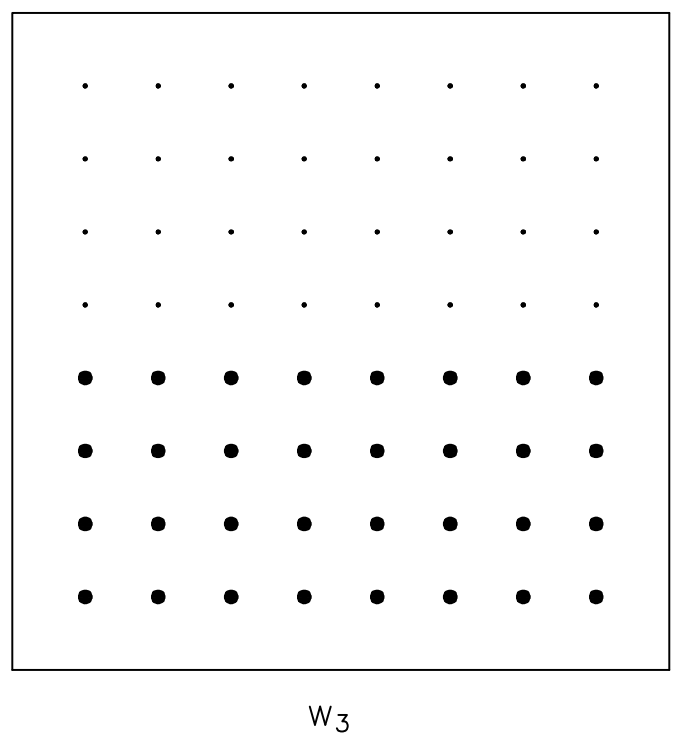

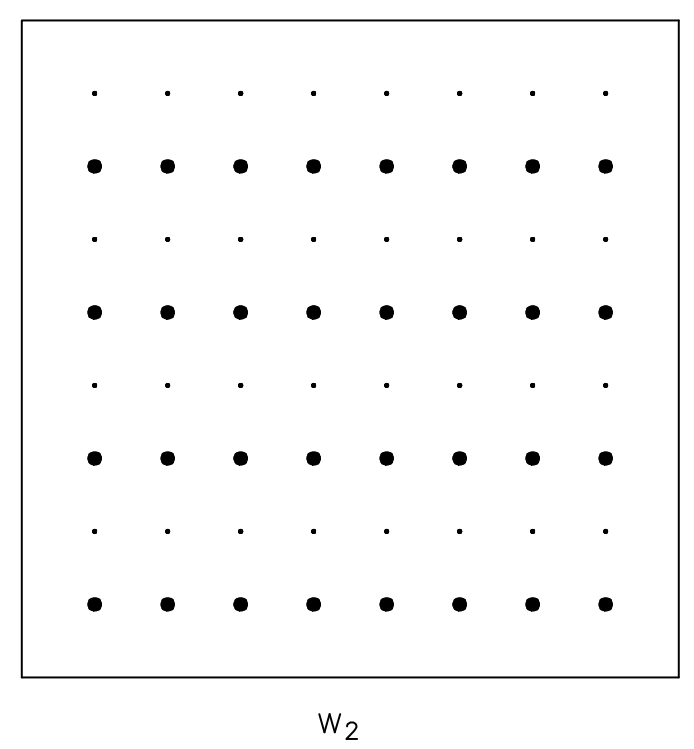

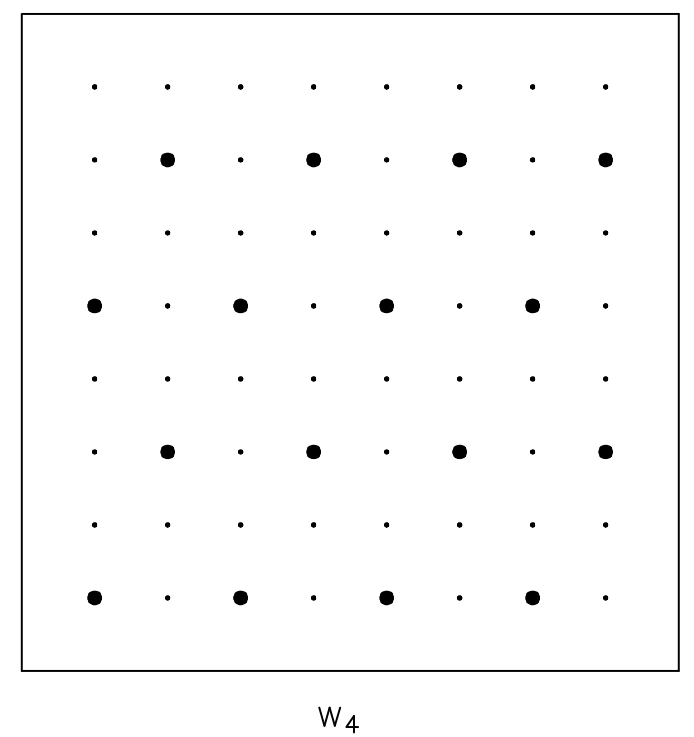

